\title{
Technical Pitfalls of Signal Truncation in Perfusion MRI of Glioblastoma
}

\author{
Kelvin K. Wong ${ }^{1,2,3 *}$, Steve H. Fung ${ }^{1,2}$, Pamela Z. New ${ }^{3}$ and Stephen T. C. Wong ${ }^{1,2}$ \\ 1 Department of Systems Medicine and Bioengineering, Houston Methodist Research Institute, Houston, TX, USA, \\ ${ }^{2}$ Department of Radiology, Weill Cornell Medicine, Cornell University, New York, NY, USA, ${ }^{3}$ Department of Neurological \\ Surgery, Weill Cornell Medicine, Cornell University, New York, NY, USA
}

\section{OPEN ACCESS}

Edited by:

Kerrie Leanne McDonald, University of New South Wales, Australia

Reviewed by: Kamalakannan Palanichamy, Ohio State University, USA David D. Eisenstat, University of Alberta, Canada

*Correspondence:

Kelvin K. Wong kwong@houstonmethodist.org

Specialty section: This article was submitted to Neuro-Oncology,

a section of the journal

Frontiers in Neurology

Received: 02 March 2016

Accepted: 20 July 2016 Published: 02 August 2016

Citation:

Wong KK, Fung SH, New PZ and Wong STC (2016) Technical Pitfalls of Signal Truncation in Perfusion MRI of Glioblastoma.

Front. Neurol. 7:121. doi: 10.3389/fneur.2016.00121
Dynamic susceptibility contrast (DSC) perfusion-weighted imaging (PWI) is widely used in clinical settings for the radiological diagnosis of brain tumor. The signal change in brain tissue in gradient echo-based DSC PWI is much higher than in spin echo-based DSC PWI. Due to its exquisite sensitivity, gradient echo-based sequence is the preferred method for imaging of all tumors except those near the base of the skull. However, high sensitivity also comes with a dynamic range problem. It is not unusual for blood volume to increase in gene-mediated cytotoxic immunotherapy-treated glioblastoma patients. The increase of fractional blood volume sometimes saturates the MRI signal during first-pass contrast bolus arrival and presents signal truncation artifacts of various degrees in the tumor when a significant amount of blood exists in the image pixels. It presents a hidden challenge in PWI, as this signal floor can be either close to noise level or just above and can go no lower. This signal truncation in the signal intensity time course is a significant issue that deserves attention in DSC PWI. In this paper, we demonstrate that relative cerebral blood volume and relative cerebral blood flow (rCBF) are underestimated due to signal truncation in DSC perfusion, in glioblastoma patients. We propose the use of second-pass tissue residue function in rCBF calculation using least-absolute-deviation deconvolution to avoid the underestimation problem.

Keywords: glioblastoma multiforme, perfusion MRI, gene therapy, MRI imaging, neuro-oncology

\section{INTRODUCTION}

Dynamic susceptibility contrast (DSC) perfusion-weighted imaging (PWI) is widely used in the radiological diagnosis of brain tumors in addition to contrast-enhanced MRI and morphological MRI. In therapeutic monitoring of brain tumors, the long standing problem is how to differentiate tumor recurrence and pseudoprogression after chemoradiation as all of these scenarios will show contrast enhancement in MRI. The literature regarding the use of perfusion to differentiate these confusing scenarios has been shown to be very useful (1-5). Machine learning-based method in the differentiation showed that perfusion measurements, such as relative cerebral blood volume (rCBV) and relative cerebral blood flow, are indeed more useful than contrast enhancement (2). Dynamic contrast enhancement (DCE) permeability mapping also showed some promise in the area (6), though it was not directly compared to DSC perfusion. The variable success in application of DSC perfusion in distinguishing progression from pseudoprogression may rest on the details of how the perfusion studies are post-processed, leading to the difficulties in comparing results across studies (7). Standardization of post-processing algorithms along with injection and imaging protocols are important issues to application of DSC perfusion in brain tumors diagnosis. 
Among the three cerebral hemodynamics parameters derived from DSC perfusion: $\mathrm{rCBV}$, relative cerebral blood flow ( $\mathrm{rCBF}$ ), and mean transit time (MTT), rCBV is the most stable, as it is computed by the area under the curve of the contrast concentration time course normalized to the area under the arterial input function (AIF). There are various methods, including deconvolution-based methods (8-10) and the tissue residue function model-based method (11), to derive rCBF and MTT. Due to the impulse response nature of the tissue residue function, which has a sharp rising edge at contrast arrival, and the limited temporal resolution, all of these methods will underestimate the peak of the tissue residue function, which leads to severe underestimation of $\mathrm{rCBF}$ at high flow rate compared to lower flow rate $(8-10)$. One way to resolve this issue is to have an accurate estimation of bolus arrival time (12), removing one parameter from the deconvolution of the tissue residue function, as a small error in the estimation of bolus arrival time can change the rCBF estimates significantly (13).

In clinical settings, the signal change in brain tissue in gradient echo DSC PWI is much higher than spin echo DSC PWI. Due to this exquisite sensitivity of gradient echo sequence, a lower contrast dose is required. Typically, spin echo DSC PWI is about half the sensitivity of gradient echo DSC PWI, with the former requiring injecting $2 \times$ the volume of contrast agent at the same concentration. Therefore, DSC PWI is a preferred method except at the base of the skull or other locations where the air-tissue interface causes significant signal dropout.

However, high sensitivity also comes with the dynamic range problem. Typical white matter and gray matter have about $2.5-5 \%$ fractional blood volume, and it is typical to tune the imaging parameters to obtain $30-60 \%$ signal drop in different brain tissues. Therefore, it is very common for the tumor signal intensity time course in gradient echo DSC PWI to drop to a noise level in pixels with a significant amount of blood volume, especially in pixels with rich venous blood or in the case of intratumoral hemorrhage. It presents a hidden challenge in PWI as the signal intensity time course can drop to a signal floor or noise floor level. In the first scenario, the average signal from the pixel is significantly above noise level because part of the pixel is occupied by a blood vessel. This signal truncation in the signal intensity time course is a significant issue that deserves attention in DSC PWI. In this paper, we demonstrate the effects of signal truncation in DSC perfusion studies of glioblastoma patients undergoing genemediated cytotoxic immunotherapy studies. The impact on CBF estimation is determined by the recirculating second pass of the tissue residue function when contrast leakage correction is used.

\section{MATERIALS AND METHODS}

\section{Theory}

To measure cerebral hemodynamic parameters, DSC perfusion image pixel intensity needs to be converted to the concentration of contrast in the tissue. The relationship between signal intensity $S(t)$ and concentration $C(t)$ is defined by:

$$
S(t)=S_{0} e^{-k C(t) \times T E}
$$

where $S_{0}$ is the image intensity before contrast injection, $k$ is a constant, and TE is the echo time. The tissue contrast concentration $C(t)$ can be expressed as a convolution of the AIF with a tissue residue function $R(t)$ multiplied by $\mathrm{CBF}$ :

$$
\begin{gathered}
C(t)=F_{t} \int_{0}^{t} \operatorname{AIF}(\tau) R(t-\tau) d \tau \\
\mathrm{MTT}=\int_{0}^{\infty} t \times h(t) d t \\
R(t)=1-\int_{0}^{t} h(t) d t
\end{gathered}
$$

where $h(t)$ is the transport function or the probability distribution of transit time in the tissue and $F_{\mathrm{t}}$ is the cerebral blood flow (CBF). The tissue contrast concentration $C(t)$ can be expressed as the convolution of the AIF, $C_{\mathrm{a}}(t)$, with a residue function $R(t)$ multiplied by CBF.

$$
\left[\begin{array}{c}
C\left(t_{0}\right) \\
C\left(t_{1}\right) \\
\vdots \\
C\left(t_{N-1}\right)
\end{array}\right]=\Delta t\left[\begin{array}{cccc}
C_{a}\left(t_{0}\right) & 0 & \cdots & 0 \\
C_{a}\left(t_{1}\right) & C_{a}\left(t_{0}\right) & \cdots & 0 \\
\vdots & \vdots & \ddots & \vdots \\
C_{a}\left(t_{N-1}\right) & C_{a}\left(t_{N-2}\right) & \cdots & C_{a}\left(t_{0}\right)
\end{array}\right] \times\left[\begin{array}{c}
R\left(t_{0}\right) \\
R\left(t_{1}\right) \\
\vdots \\
R\left(t_{N-1}\right)
\end{array}\right] F_{\mathrm{t}}
$$

which can be simplified to $c=A \cdot r$ and CBF can be estimated as the maximum value of $r$.

The CBF rate can be estimated by a perfusion deconvolution algorithm using least-absolute-deviation (LAD) regularization (10), which allows more accurate estimation at high blood volume and high flow rate. LAD regularization can be achieved by minimizing the following functional:

$$
r_{\lambda}=\min \left\{{ }_{r}\left\{\|A r-c\|_{1}+\lambda\|L r\|_{1}\right\}\right.
$$

where L1 norm is defined as $\|x\|_{1}=\sum_{i=1}^{n}\left|x_{i}\right|$; the regularization function $L$ is chosen as a first order difference operator and $\lambda$ is the regularization parameter. The minimization problem is formulated as a linear programing problem and solved iteratively by the interior point method (14).

Note that the ideal tissue residue function $R(t)$ in the absence of recirculation is by definition a monotonic decay function, such as exponential, boxcar, or triangular functions (10). In the presence of second-pass recirculation, the measured tissue residue function $R_{\mathrm{m}}(t)$ is a summation of the tissue residue function of the first pass and the second pass with a time-shift corresponding to the vascular delay $\left(V_{\mathrm{d}}\right)$ and a Gaussian-like vascular dispersion function $[D(t)]$ caused by the recirculation system.

$$
R_{\mathrm{m}}(t)=R(t)+D(t) \otimes R\left(t-V_{\mathrm{d}}\right)
$$

Assuming the first-pass tissue residue function does not extend beyond $V_{\mathrm{d}}$, which is a reasonable assumption in brain tissue, as $V_{\mathrm{d}}$ is at least $20 \mathrm{~s}$ in typical adult with normal cardiac output, $\mathrm{rCBF}$ can be determined as the peak of the second-pass tissue residue 
function, as the same dispersion function applies linearly on all pixels in tissue.

\section{Leakage Correction in Brain Tumor}

Contrast leakage is expected to occur in most brain tumors and it is also one of the fundamental criteria to detect the spatial extent of brain tumors, clinically. Some brain tumors are non-enhancing though glioblastoma are mostly enhancing tumors with some non-enhancing components. In the presence of contrast leakage to parenchyma in strongly $\mathrm{T} 2{ }^{\star}$-weighted PWI, the measured contrast concentration in tissue, $C_{\mathrm{m}}(t)$, calculated using Eq. 1 can be effectively modeled as (15):

$$
C_{\mathrm{m}}(t)=K_{1} C_{\text {tissue }}(t)+K_{2} \int_{0}^{t} C_{\text {tissue }}(t)
$$

where $C_{\text {tissue }}(t)$ is the mean contrast concentration curve of normal brain tissue including both gray and white matter, $K_{2}$ is a heuristic factor primarily determined by the rate of leakage, such as permeability surface area product, etc. The leakage corrected tissue contrast concentration $C(t)$ is thus equal to $K_{1} C_{\text {tissue }}(t)$.

\section{Clinical Cases}

To demonstrate the signal truncation effects in rCBF measurements, longitudinal data from glioblastoma patients in gene-mediated cytotoxic immunotherapy studies (16) (www. ClinicalTrials.gov; ID: NCT00751270 and NCT00589875) were retrieved from the picture archiving system, retrospectively. The DSC perfusion study was conducted in a General Electric 1.5 T/3.0 T Signa Excite system using a 8-channel head coil. The imaging parameters of the $1.5 \mathrm{~T}$ protocol are: TR/TE: 1400$1700 \mathrm{~ms} / 60 \mathrm{~ms}, 2 \mathrm{D}$ single-shot gradient-echo EPI sequence with $7 \mathrm{~mm}$ slice thickness with in-plane resolution of $1.875 \mathrm{~mm}$ and the 3.0 T protocol parameters are TR/TE: $1600-2000 \mathrm{~ms} / 40 \mathrm{~ms}, 2 \mathrm{D}$ single shot gradient-echo EPI sequence with $5 \mathrm{~mm}$ slice thickness with same in-plane resolution. Some cases were conducted with a power injector where $15 \mathrm{~mL}$ contrast agent is injected at $5 \mathrm{~mL} / \mathrm{s}$, intravenously. The study was approved by the institutional review board of the Houston Methodist Hospital.

\section{RESULTS}

Figure 1 shows the $\mathrm{rCBV}$ and $\mathrm{rCBF}$ maps from the first-pass and second-pass tissue residue function of a representative $3 \mathrm{~T}$ case done with a power injector. Note the close resemblance of the $\mathrm{rCBV}$ and $\mathrm{rCBF}$ map generated with the second-pass tissue residue function, indicating a tight coupling. Figure 2 shows the truncation artifacts on the single-pixel signal time course within the time window of [16 s, $34 \mathrm{~s}$ ] when the contrast bolus pass through different pixel locations. The mean noise level was about $100 \mathrm{au}$. Various forms of signal truncation are shown at Locations 1,3 , and 4 , while Location 2 is not truncated. The truncation issue is very common in areas with high blood volume in tumor tissue, especially near blood vessels. Figure 3 shows the effect of signal truncation on the tissue residue function. The first-pass tissue residue function is essentially truncated near the top, which will significantly underestimate $\mathrm{rCBF}$. The first-pass and second-pass portion of the tissue residue function are well separated, and the second-pass portion of the tissue residue function has a monotonic decay shape after the peak.

Figure 4 shows the rCBV and $\mathrm{rCBF}$ maps from the first-pass and second-pass tissue residue function of a representative $1.5 \mathrm{~T}$ case done without using a power injector. The post-contrast T1-enhanced region overlapped spatially with high $\mathrm{rCBV}$ and rCBF regions either using the peak of the first-pass or the secondpass tissue residue function. This is the worst-case scenario where manual injection was not fast enough to allow a clear separation between first-pass and second-pass bolus passage. It also violates the assumptions of a separable second-pass. Nevertheless, Figure 5 shows the effect of signal truncation and the eventual distortion on the contrast concentration time courses. The leakage correction still performs fine as the contrast concentration returns to very low level at steady-state in the presence of small signal truncation error.

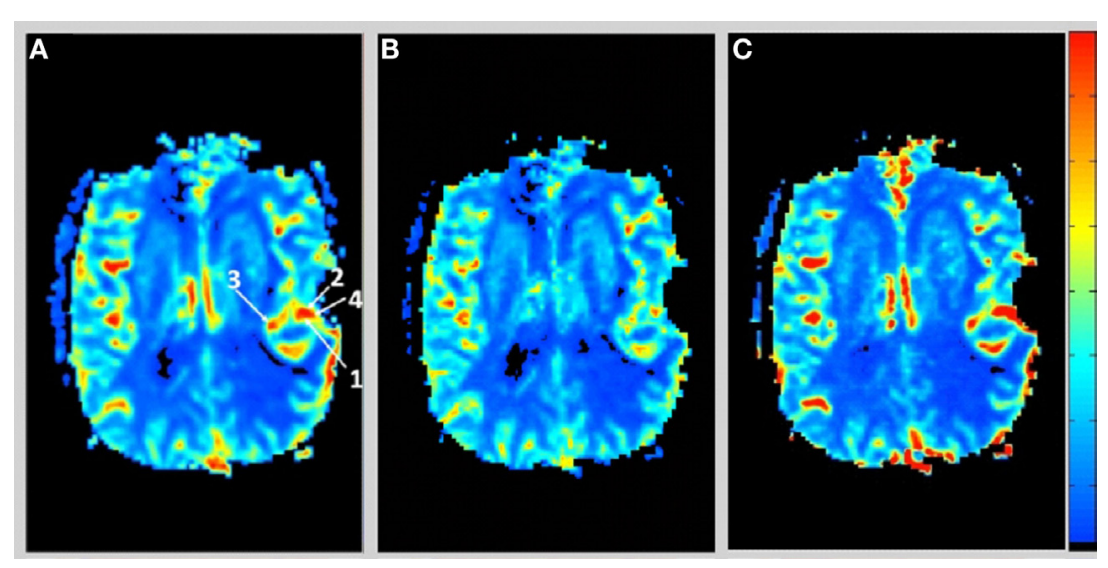

FIGURE 1 | (A) Relative cerebral blood volume map generated from the leakage corrected data, (B) relative cerebral blood flow map determined by the peak of the first-pass tissue residue function, and $\mathbf{( C )}$ relative cerebral blood flow map determined by the peak of the second-pass tissue residue function. Note the tight coupling between blood volume and blood flow. 


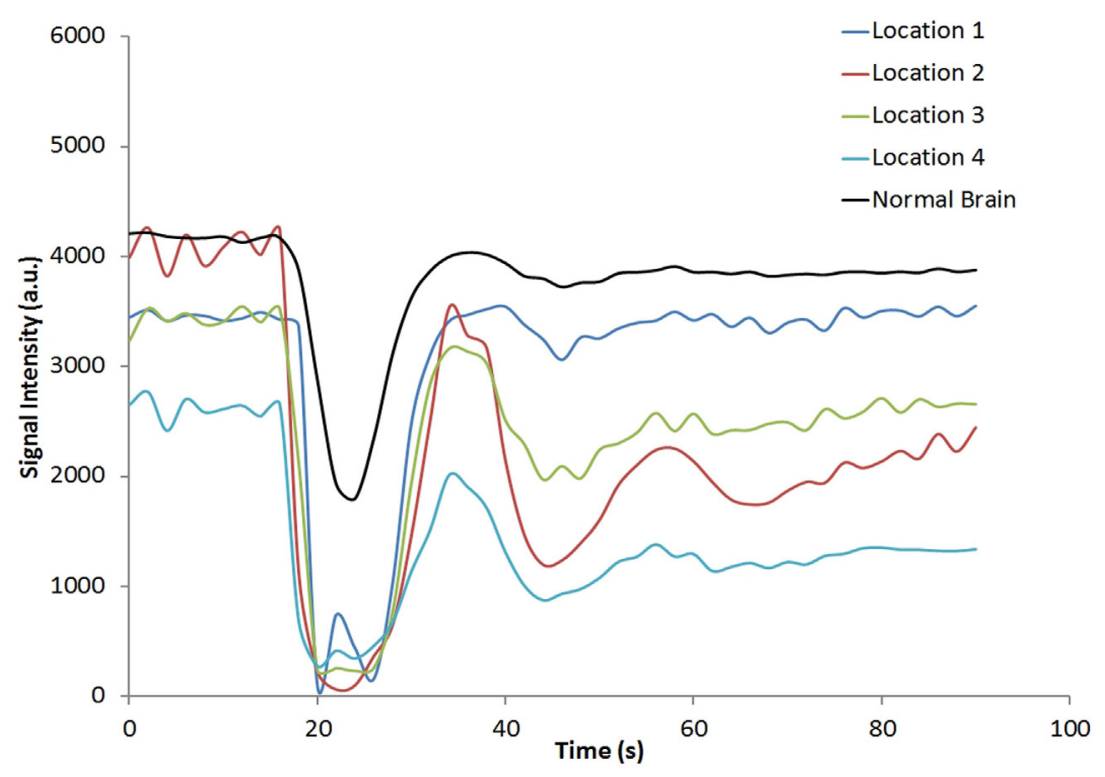

FIGURE 2 | Signal intensity time courses of different high blood volume locations and in normal brain tissue. All locations except Location 2 have signal truncation artifacts due to high blood volume. Their respective locations are indicated by arrows in Figure 1.

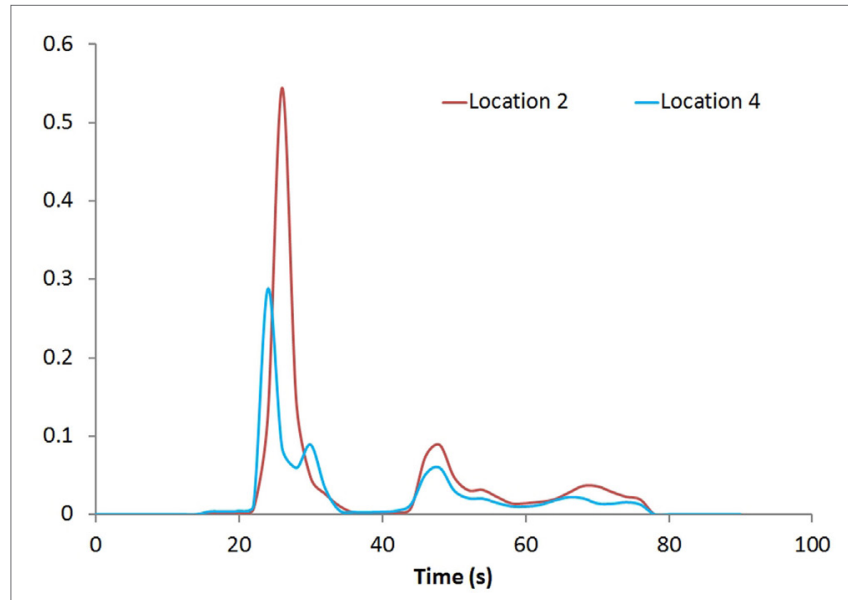

FIGURE 3 | Tissue residue functions at Locations 2 and $\mathbf{4}$. Both the first-pass [20s, 40s] and second-pass [40s, 60s] of the function are well separated.

\section{DISCUSSION}

One factor that limits our ability to quantify $\mathrm{CBF}, \mathrm{CBV}$, and MTT from DSC perfusion studies is the necessity of measuring the signal in the artery (i.e., AIF) that feeds the tissue of interest, whose shape depends on injection speed, cardiac output, etc. It has been shown that errors in selecting the AIF can be among the largest sources of error in the derived measurements of CBV, CBF, and MTT. Truncation of the AIF shape has a profound effect on the maps generated.
Truncation of the tissue signal time course also has a profound effect on the maps generated. This problem is less addressed in the literature as the effect is quite different on a pixel-to-pixel basis depending on the severity of truncation. The various shapes and form of truncation can be hard to recognize as saturation of contrast agent in part of a pixel is not equivalent to signal hitting the floor as partial volume effect determines the ultimate signal behavior. Image pixels with a large blood-containing fraction are more likely to have signal truncation artifacts. Truncation in the AIF can be mitigated to some extent by fitting the firstpass portion of the AIF using the gamma-variate function (17), which is also used in this study. Truncation in the tissue level is, however, difficult to compensate as the tissue residue function is unknown, and it can greatly affect the shape and form of the contrast concentration curves.

The proposed extension to the LAD deconvolution method works well in brain tumor cases that are based on the assumption that there is little overlap between the first-pass and second-pass portions of the tissue residue function. It applies generally where the contrast bolus is injected quickly with the help of a power injector where the first-pass and second-pass contrast passage are well separated. In tissue ischemia, where the MTT is very long, i.e., the tissue residue function has a long tail, there will be significant overlaps between the first-pass and second-pass portion. This limits application in tissue with known long transit time, such as choroid plexus, or in ischemic tissue. Note that this assumption is implicitly used in leakage correction, which models that the contrast concentration time course in tumor tissue is proportional to the contrast concentration time course of normal tissue. Therefore, leakage correction should be used and interpreted with caution near tissue with long transit time in brain tumor cases. 


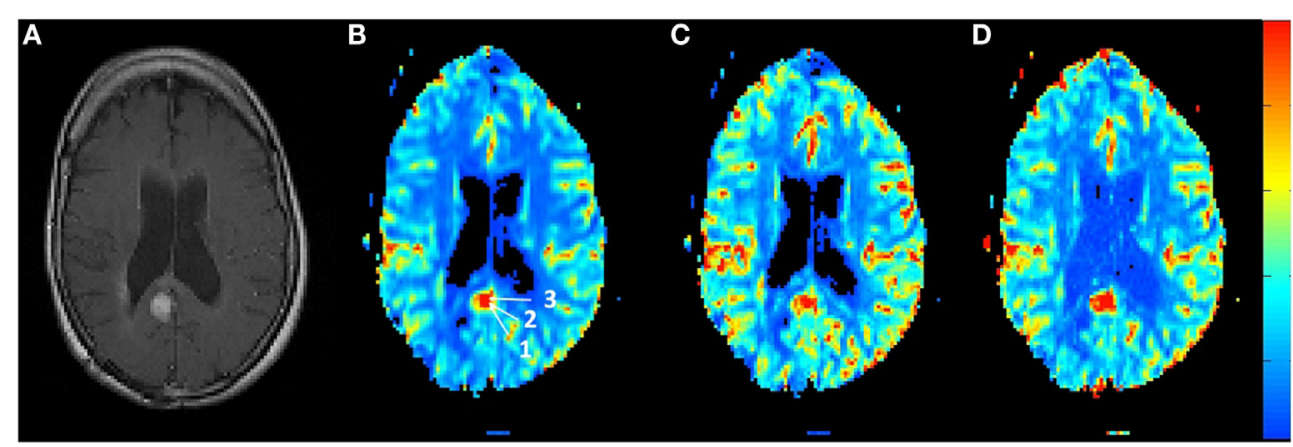

FIGURE 4 | (A) T1-weighted post-contrast MRI showing the location of suspected tumor. The suspected enhanced region has a hot spot area in the (B) relative blood volume map as well as in the (C) relative cerebral blood flow map determined by the peak of the first-pass tissue residue function as well from the (D) secondpass tissue residue function.

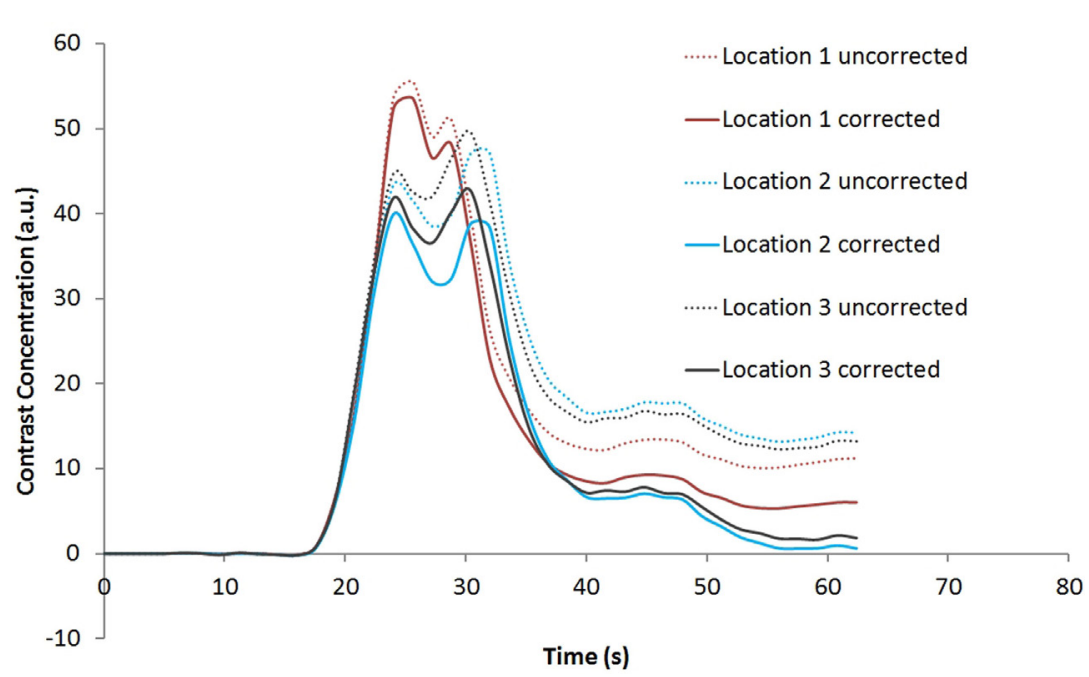

FIGURE 5 | Contrast concentration time courses of Locations 1-3 indicated in Figure 4B before and after contrast leakage correction. Signal truncation artifact at these locations results in a disruption of the contrast concentration time course. The effect of leakage correction is obvious with minimal contrast concentration after the first and second passes of the bolus.

In addition, we would like to point out that, in LAD deconvolution of tissue residue function, temporal signal-to-noise ratio and the temporal contrast change during bolus passage are two important factors for the method to work properly rather than static image signal-to-noise ratios. To quantify the tissue residue function in more detail, one would need higher temporal resolution while simultaneously addressing the accompanying T1 leakage effects in tumor cases (18). Table $\mathbf{1}$ shows a summary of the technical pitfalls and potential solutions discussed in this paper.

One notable effect of using the second-pass tissue residue function in determining $\mathrm{rCBF}$ is that the major veins are suppressed. This is obvious in the suppression of sagittal sinus in Figure 1C compared to Figure 1B. This is understandable as major veins effectively collect the blood after the passage of the bolus through the whole brain and have significantly higher vascular dispersion. Sagittal sinus is the most suppressed. It is
TABLE 1 | Summary of technical pitfalls and potential solutions.

\begin{tabular}{ll}
\hline Pitfalls & Solutions \\
\hline $\begin{array}{l}\text { Arterial input function } \\
\text { truncation (AIF) }\end{array}$ & Fitting AIF with a Gamma-variate function \\
$\begin{array}{l}\text { Brain tissue signal time } \\
\text { course truncation }\end{array}$ & $\begin{array}{l}\text { 1. Leakage correction } \\
\text { 2. Calculate rCBF and rCBV using second- } \\
\text { pass tissue residue function }\end{array}$
\end{tabular}

also noteworthy that, in the calculation of rCBV, signal truncation artifacts in major veins will also cause a truncation in the contrast concentration time curve, leading to underestimation of rCBV. This may have significant implications in tumor rCBV interpretation. The underestimation of $\mathrm{rCBV}$ due to signal truncation is less severe than the case in $\mathrm{rCBF}$. It has been shown that $\mathrm{rCBV}$ and $\mathrm{rCBF}$ are two of the most important parameters 
to differentiate pseudoprogression and tumor recurrence in glioblastoma patients, after chemoradiation $(2,4-6)$. Radiation necrosis is associated with low rCBV and rCBF. Gross underestimation of $\mathrm{rCBV}$ and $\mathrm{rCBF}$ due to signal truncations would bias the diagnosis toward pseudoprogression, systematically. However, perfusion change during immunotherapy in glioblastoma could be therapy-specific, and more studies are needed to understand the role of perfusion imaging to monitor disease progression.

To summarize, we propose the use of second-pass tissue residue function in $\mathrm{rCBF}$ calculation using LAD deconvolution method. This strategy is used to circumvent the practical limitations in DSC PWI, where signal truncation artifacts occur often in high blood volume regions. The issue has not received much attention in the literature, and the problem is more severe in high-grade brain tumor where the rCBV is very high. Implications of signal truncation in high blood volume regions leading to underestimation in $\mathrm{rCBV}$ and $\mathrm{rCBF}$ are discussed. In the absence of severe

\section{REFERENCES}

1. Boxerman JL, Ellingson BM, Jeyapalan S, Elinzano H, Harris RJ, Rogg JM, et al. Longitudinal DSC-MRI for distinguishing tumor recurrence from pseudoprogression in patients with a high-grade glioma. Am J Clin Oncol (2014). doi:10.1097/COC.0000000000000156

2. Hu X, Wong KK, Young GS, Guo L, Wong ST. Support vector machine multiparametric MRI identification of pseudoprogression from tumor recurrence in patients with resected glioblastoma. J Magn Reson Imaging (2011) 33:296-305. doi:10.1002/jmri.22432

3. Choi YJ, Kim HS, Jahng GH, Kim SJ, Suh DC. Pseudoprogression in patients with glioblastoma: added value of arterial spin labeling to dynamic susceptibility contrast perfusion MR imaging. Acta Radiol (2013) 54:448-54. doi:10.1177/0284185112474916

4. Gahramanov S, Muldoon LL, Varallyay CG, Li X, Kraemer DF, Fu R, et al. Pseudoprogression of glioblastoma after chemo- and radiation therapy: diagnosis by using dynamic susceptibility-weighted contrast-enhanced perfusion MR imaging with ferumoxytol versus gadoteridol and correlation with survival. Radiology (2013) 266:842-52. doi:10.1148/radiol.12111472

5. Young RJ, Gupta A, Shah AD, Graber JJ, Chan TA, Zhang Z, et al. MRI perfusion in determining pseudoprogression in patients with glioblastoma. Clin Imaging (2013) 37:41-9. doi:10.1016/j.clinimag.2012.02.016

6. Thomas AA, Arevalo-Perez J, Kaley T, Lyo J, Peck KK, Shi W, et al. Dynamic contrast enhanced T1 MRI perfusion differentiates pseudoprogression from recurrent glioblastoma. J Neurooncol (2015) 125:183-90. doi:10.1007/ s11060-015-1893-Z

7. Hu LS, Kelm Z, Korfiatis P, Dueck AC, Elrod C, Ellingson BM, et al. Impact of software modeling on the accuracy of perfusion MRI in glioma. AJNR Am J Neuroradiol (2015) 36(12):2242-9. doi:10.3174/ajnr.A4451

8. Wu O, Østergaard L, Weisskoff RM, Benner T, Rosen BR, Sorensen AG. Tracer arrival timing-insensitive technique for estimating flow in MR perfusion-weighted imaging using singular value decomposition with a block-circulant deconvolution matrix. Magn Reson Med (2003) 50:164-74. doi: $10.1002 / \mathrm{mrm} .10522$

9. Smith MR, Lu H, Trochet S, Frayne R. Removing the effect of SVD algorithmic artifacts present in quantitative MR perfusion studies. Magn Reson Med (2004) 51:631-4. doi:10.1002/mrm.20006

10. Wong KK, Tam CP, Ng M, Wong ST, Young GS. Improved residue function and reduced flow dependence in MR perfusion using least-absolute-deviation regularization. Magn Reson Med (2009) 61:418-28. doi:10.1002/mrm.21860 tissue ischemia, $\mathrm{rCBF}$ maps derived from second-pass tissue residue function may be a useful alternative. By paying attention to the technical pitfalls described in this paper, $\mathrm{rCBV}$ and $\mathrm{rCBF}$ underestimations can be avoided. Unbiased estimations of $\mathrm{rCBF}$ and $\mathrm{rCBV}$ are crucial to differentiate pseudoprogression from tumor recurrence.

\section{AUTHOR CONTRIBUTIONS}

KW designed, conducted the studies, and prepared the manuscript. SF and PN provided clinical data and contributed to manuscript preparation. SW contributed to manuscript preparation.

\section{FUNDING}

KW is supported by R01CA185402, Golfer Against Cancer and TT and WF Chao Foundation. KW is supported by TT and WF Chao Foundation and John S. Dunn Research Foundation.

11. Mehndiratta A, MacIntosh BJ, Crane DE, Payne SJ, Chappell MA. A control point interpolation method for the non-parametric quantification of cerebral haemodynamics from dynamic susceptibility contrast MRI. Neuroimage (2013) 64:560-70. doi:10.1016/j.neuroimage.2012.08.083

12. Wong K, Tam C, Ng M. Accurate vascular delay estimation from low-temporal resolution image data set [life sciences]. IEEE Signal Process Mag (2010) 27:155-8. doi:10.1109/MSP.2010.936059

13. Ibaraki M, Shimosegawa E, Toyoshima H, Takahashi K, Miura S, Kanno I. Tracer delay correction of cerebral blood flow with dynamic susceptibility contrast-enhanced MRI. JCereb Blood Flow Metab (2005) 25:378-90. doi:10.1038/sj.jcbfm.9600037

14. Gruner R, Taxt T. Iterative blind deconvolution in magnetic resonance brain perfusion imaging. Magn Reson Med (2006) 55:805-15. doi:10.1002/ mrm. 20850

15. Boxerman JL, Schmainda KM, Weisskoff RM. Relative cerebral blood volume maps corrected for contrast agent extravasation significantly correlate with glioma tumor grade, whereas uncorrected maps do not. AJNR Am J Neuroradiol (2006) 27:859-67.

16. Chiocca EA, Aguilar LK, Bell SD, Kaur B, Hardcastle J, Cavaliere R, et al. Phase IB study of gene-mediated cytotoxic immunotherapy adjuvant to up-front surgery and intensive timing radiation for malignant glioma. J Clin Oncol (2011) 29:3611-9. doi:10.1200/JCO.2011.35.5222

17. Patil V, Johnson G. An improved model for describing the contrast bolus in perfusion MRI. Med Phys (2011) 38:6380-3. doi:10.1118/1.3658570

18. Liu HL, Wu YY, Yang WS, Chen CF, Lim KE, Hsu YY. Is Weisskoff model valid for the correction of contrast agent extravasation with combined T1 and T2* effects in dynamic susceptibility contrast MRI? Med Phys (2011) 38:802-9. doi:10.1118/1.3534197

Conflict of Interest Statement: The authors declare that the research was conducted in the absence of any commercial or financial relationships that could be construed as a potential conflict of interest.

Copyright (c) 2016 Wong, Fung, New and Wong. This is an open-access article distributed under the terms of the Creative Commons Attribution License (CC BY). The use, distribution or reproduction in other forums is permitted, provided the original author(s) or licensor are credited and that the original publication in this journal is cited, in accordance with accepted academic practice. No use, distribution or reproduction is permitted which does not comply with these terms. 